\title{
ESTUDO DA VAZÃO E CONSTRUÇÃO DE CENÁRIOS A PARTIR DAS MUDANÇAS DE USO DA TERRA E COBERTURA VEGETAL NATIVA DA BACIA HIDROGRÁFICA DO ALTO CURSO DO RIO MISERICÓRDIA - MG
}

\author{
Mirna Karla Amorim da Silva ${ }^{(a)}$, Roberto Rosa $^{(b)}$ \\ (a) Instituto de Geografia, Universidade Federal de Uberlândia, mirnakarla@ufu.br \\ (b) Instituto de Geografia, Universidade Federal de Uberlândia, rrosa@ufu.br
}

Eixo: GEOTECNOLOGIAS E MODELAGEM ESPACIAL EM GEOGRAFICA FÍSICA

\begin{abstract}
Resumo
Esta pesquisa teve como objetivo estudar a vazão e construir cenários a partir das mudanças de uso da terra e cobertura vegetal nativa, na bacia hidrográfica do alto curso do rio Misericórdia, localizada em parte dos municípios de Ibiá, Pratinha e Campos Altos, Minas Gerais. A principal ferramenta utilizada foi o modelo hidrológico MGB-IPH. As vazões mínima, máxima, média e o escoamento total anual foram analisados em cenário real (2010) e cenários hipotéticos de uso da terra. De forma geral, verificou-se que a substituição da cobertura vegetal nativa pelo uso da pastagem, aumentou a vazão máxima $\left(76,27\right.$ para $\left.105,72 \mathrm{~m}^{3} / \mathrm{s}\right)$ e a vazão média $\left(23,98\right.$ para $\left.24,74 \mathrm{~m}^{3} / \mathrm{s}\right)$ e reduziu a vazão mínima $\left(10,18\right.$ para $\left.6,19 \mathrm{~m}^{3} / \mathrm{s}\right)$. A substituição da cobertura vegetal nativa pela agricultura resultou em redução da vazão máxima $\left(76,27\right.$ para $\left.47,28 \mathrm{~m}^{3} / \mathrm{s}\right)$, mínima $\left(10,18\right.$ para $\left.9,25 \mathrm{~m}^{3} / \mathrm{s}\right)$ e da vazão média $\left(23,98\right.$ para $\left.19,24 \mathrm{~m}^{3} / \mathrm{s}\right)$.
\end{abstract}

Palavras chave: Modelagem hidrológica. Uso da terra. Vazão.

\section{Introdução}

A água como um elemento básico da vida, deve ser cuidada e preservada para a atual e futuras gerações. No entanto, verifica-se um grande consumo de água pela agricultura, indústria e pelas pessoas, o que acarreta altos custos ambientais, incluindo a perda de biodiversidade e danos aos sistemas hídricos naturais (rios e aquíferos).

Neste sentido, a temática sobre modelagem ambiental, a exemplo da modelagem hidrológica, vem sendo amplamente estudada e contribui de maneira relevante na preservação do meio ambiente. A modelagem hidrológica permite estimar as transformações hidrológicas ocorridas em bacias hidrográficas, especialmente advindas de cenários de mudanças de uso da terra e cobertura vegetal nativa, a partir de dados espaciais e geotecnologias disponíveis.

Dentro desta temática, as bacias hidrográficas são consideradas como unidade básica de planejamento e gerenciamento ambiental comumente utilizadas, conforme pesquisas de estudiosos como Rocha (1991) e 
Tundisi e Tundisi (2005). Chaudhry, Paiva e Reis (2004) destacaram que a bacia hidrográfica é considerada como referência espacial e elemento fundamental de análise do estudo dos processos hidrodinâmicos e os problemas relacionados à modificação de suas características naturais assumem importância socioeconômica cada vez maior.

Diversos são os estudos realizados em bacias hidrográficas e a modelagem matemática está presente na prática hidrológica como uma das principais ferramentas utilizadas em avaliações ambientais. De forma geral, os modelos surgem no sentido de auxiliar o entendimento dos processos ocorridos na natureza a partir de dados de entrada conhecidos para a formulação/verificação de resultados desconhecidos.

Desta maneira, a modelagem hidrológica surgiu para otimizar o gerenciamento dos recursos hídricos, avaliar os impactos de mudança do uso da terra e cobertura vegetal nativa e das mudanças climáticas, alertar quanto à operação de sistemas de recursos hídricos e a ocorrência de cheias, analisar a consistência e extensão de séries hidrológicas em locais com poucas informações, entre outros. Bayer e Collischonn (2013) mostraram, como exemplo, a utilização dos modelos hidrológicos para o estudo da alteração nas medidas de vazão em cursos d'água em locais que tiveram reduções de áreas florestadas.

Christofoletti (1999) realçou a importância do uso dos Sistemas de Informação Geográficas (SIG’s) nos processos de análise espacial e modelagens. Em estudos hidrológicos, especificamente, o Sensoriamento Remoto se destaca como fonte de aquisição de dados e os SIG's, como ferramenta na aquisição e manipulação dos dados espaciais e/ou temporais, análises e aplicação de modelagens hidrológicas.

De acordo com Rennó e Soares (2007, p. 531) "um modelo hidrológico pode ser definido como uma representação matemática do fluxo de água e seus constituintes sobre alguma parte da superfície e/ou subsuperfície terrestre". Deste modo, modelar o comportamento dos fenômenos hidrológicos permite testar e avaliar diferentes formulações para os processos hidrológicos analisados em uma bacia hidrográfica.

Os modelos hidrológicos têm importante papel na simulação de situações hipotéticas para conhecimento do comportamento dos processos hidrológicos. Além de permitir diversas simulações em cenários hipotéticos e/ou futuros de forma confiável, a modelagem hidrológica pode ser utilizada de modo rápido e com menores custos de execução em relação às pesquisas experimentais. Os resultados desse tipo de simulação permitem conhecer as consequências de determinado tipo de uso e manejo em uma bacia hidrográfica e a resposta daquele ambiente a uma determinada situação. No entanto, não deixando de se considerar que outros fatores, especialmente em diferentes escalas, estejam associados ao comportamento hidrológico de um rio, a exemplo das mudanças climáticas, da topografia da bacia, etc. 


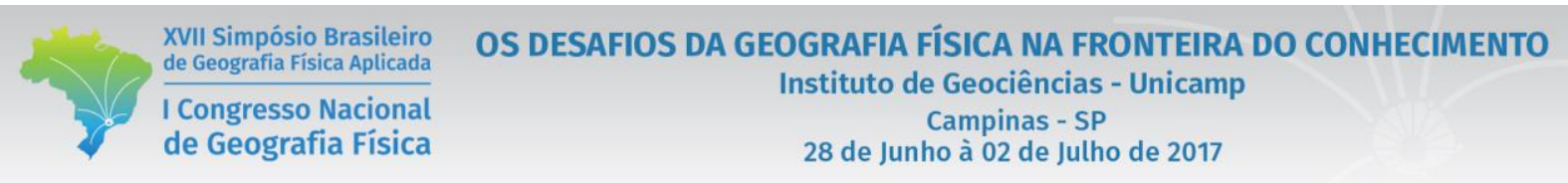

Diversos modelos hidrológicos podem ser encontrados na literatura. Nesta pesquisa foi utilizado o MGB, Modelo de Grandes Bacias, desenvolvido no Instituto de Pesquisas Hidráulicas (IPH) da Universidade Federal do Rio Grande do Sul (UFRGS). O MGB é um modelo matemático apresentado nos estudos de Collischonn (2001), que apresentou o desenvolvimento e a validação deste modelo hidrológico.

Definidos todos os parâmetros necessários e calibrado corretamente, o MGB permite o estudo do comportamento de diferentes componentes do sistema hidrológico, sendo capaz de estimar séries de vazão a partir de dados de precipitação, prever a vazão de cheias e avaliar o seu impacto, estimar os efeitos de cenários futuros do uso da terra, alterações climáticas e uso dos recursos hídricos nas bacias em estudo, entre várias outras aplicações, no sentido de avançar as técnicas e metodologias de previsão hidrológica.

Assim, a modelagem de cenários futuros de planejamento é uma ferramenta importante dentro da modelagem hidrológica e está condicionada aos parâmetros de entrada e fatores de ajuste do modelo, pois, conhecendo-se tais parâmetros e o risco de ocorrência dos mesmos, é possível estimar cenários visando o dimensionamento e/ou planejamento de alternativas de desenvolvimento para o sistema.

Neste contexto, esta pesquisa teve como objetivo estudar a vazão e construir cenários a partir de mudanças do uso da terra e cobertura vegetal nativa. Como estudo de caso, foi escolhida da bacia hidrográfica do alto curso do rio Misericórdia, localizada em parte dos municípios de Ibiá, Pratinha e Campos Altos, no Estado de Minas Gerais, com área de 1.372,30 km² (Figura 1). O rio Misericórdia é afluente do rio Quebra-anzol, que desagua no rio Araguari, rio este que compõe uma importante bacia hidrográfica da Mesorregião do Triângulo Mineiro e Alto Paranaíba.

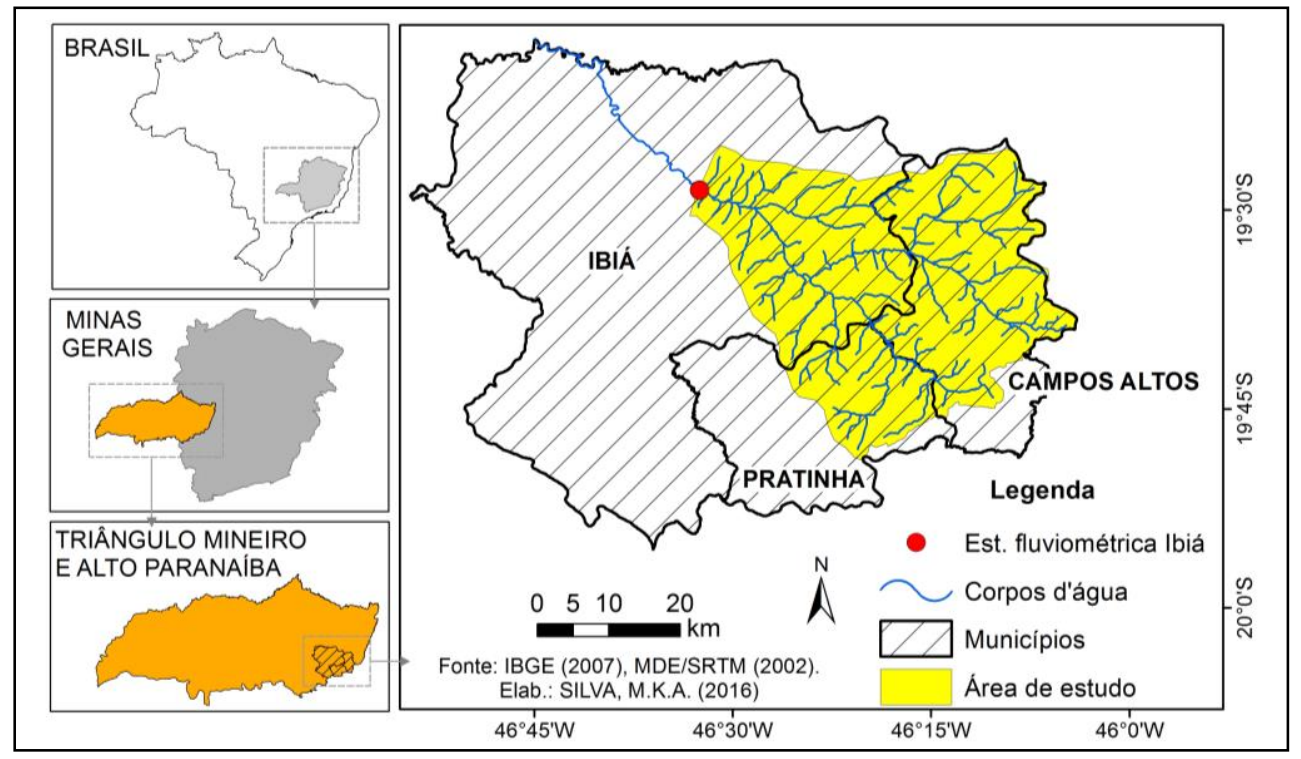

Figura 1 - Mapa de localização geográfica da área de estudo 


\section{Materiais e procedimentos operacionais}

Esta pesquisa utilizou uma série de dados espaciais (imagens de satélite, mapas temáticos, cartas topográficas, etc.) e, ainda, dados quantitativos (climáticos e fluviométricos), a partir de órgãos que disponibilizam gratuitamente esse material. Dentre eles:

- Dados vetoriais de classificação de solos, geomorfologia e geologia da Bacia Hidrográfica do Rio Araguari (CBH-ARAGUARI, 2013);

- Dados vetoriais do uso da terra e cobertura vegetal nativa da Bacia Hidrográfica do Rio Araguari (ROSA, 2012), ajustados a partir de imagens do sensor TM/Landsat 5, para o ano de 2010;

- Modelo Digital de Elevação, obtido pela missão SRTM (2002);

- Dados hidrológicos diários (vazão, chuva, temperatura, umidade relativa, velocidade do vento, insolação, pressão atmosférica) disponibilizados pelas séries históricas do banco de dados da Agência Nacional de Águas (ANA, 2013).

Foram utilizados os softwares ArcGIS 10.1, MapWindows GIS e o aplicativo de Manejo de dados Hidroweb.

Após a coleta dos dados iniciais, foram elaborados alguns mapas temáticos necessários para a pesquisa, dentre eles: solos, unidades de relevo, geológico, potencial de geração de escoamento superficial e uso da terra e cobertura vegetal nativa (2010).

Posteriormente, foi realizada a preparação para aplicação do MGB, de acordo com os tutoriais disponibilizados pela própria equipe de desenvolvimento do modelo, da UFRGS, a partir da URL: $<$ http://www.ufrgs.br/hge/mgb-iph> (UFRGS, 2011).

Foi realizada a discretização da bacia em mini-bacias para sua correta e mais detalhada utilização no modelo hidrológico, a determinação das unidades de resposta hidrológica (combinações de tipo de solo, com a geologia, geomorfologia e uso da terra na área de estudo), a preparação dos dados de entrada do PrePro-MGB (rede de drenagem, mini-bacias, etc.) e dos dados de entrada do MGB (dados climáticos, parâmetros fixos e calibráveis, etc.), com o auxílio do MapWindow. O MGB foi calibrado (calibração manual e automática) e, posteriormente, foram realizadas as simulações necessárias.

Para a simulação da vazão nos cenários hipotéticos da bacia, foi necessário realizar ajustes nos parâmetros do modelo que são importantes para diferenciar usos da terra e cobertura vegetal nativa: o albedo, o índice de área foliar, a resistência aerodinâmica e a resistência superficial, além dos parâmetros Wm 
(armazenamento máximo na camada superficial do solo, em mm) e b (relação empírica entre fração de área saturada e armazenamento médio de unidade do solo), que também devem ser alterados de acordo com os tipos de uso da terra e cobertura vegetal nativa presentes na área de estudo.

A vazão da bacia foi simulada em cenário real de uso da terra (2010) e em quatro diferentes cenários hipotéticos, conforme Tabela I.

Tabela I - Cenários hipotéticos simulados para a bacia hidrográfica do alto curso do rio Misericórdia

\begin{tabular}{c}
\hline Cenários \\
\hline $100 \%$ Cobertura vegetal nativa \\
$100 \%$ Pastagem \\
$100 \%$ Agricultura \\
Conversão da pastagem para agricultura \\
Elaboração: A autora.
\end{tabular}

A partir das simulações realizadas com os cenários hipotéticos propostos, foram verificados as vazões mínimas, máximas, médias e o valor do escoamento total anual, para a bacia em estudo. Foram comparadas as vazões simuladas (cenários hipotéticos) em relação a vazão correspondente ao ano de 2010 (cenário real).

\section{Resultados e discussões}

Apesar de não ser o único processo responsável pela alteração do comportamento de vazão de um rio, a mudança de uso da terra afeta o balanço entre a precipitação e a evaporação e, consequentemente, influencia no comportamento da vazão nos cursos d'água.

Para o MGB, a sensibilidade de alguns parâmetros específicos é importante na análise das consequências dessas mudanças de uso na resposta hidrológica dos cursos d'água, como já comentado anteriormente. A definição de valores confiáveis para estes parâmetros determina a confiabilidade dos resultados para as simulações realizadas em cenários de mudança de uso da terra e cobertura vegetal nativa. Assim, considerando válidos os parâmetros que influenciam diretamente na análise das categorias de uso da terra e cobertura vegetal nativa, definidos para a simulação da bacia em estudo, o MGB foi utilizado para realizar a simulação de cenários hipotéticos de mudança de uso e cobertura vegetal nativa a fim de verificar qual seria o comportamento da vazão, no rio Misericórdia, em situações diversas da realidade. 


\section{OS DESAFIOS DA GEOGRAFIA FÍSICA NA FRONTEIRA DO CONHECIMENTO \\ Instituto de Geociências - Unicamp \\ Campinas - SP \\ 28 de Junho à 02 de Julho de 2017}

No ano de 2010 foi possível verificar a distribuição de área das classes de uso da terra e cobertura vegetal nativa, conforme Tabela II.

Tabela II - Área das classes de uso da terra e cobertura vegetal nativa da bacia em estudo (2010)

\begin{tabular}{l|rr}
\hline \multicolumn{1}{c|}{ Classe } & \multicolumn{2}{c}{$\mathbf{2 0 1 0}$} \\
\cline { 2 - 3 } & $\mathbf{k m}^{\mathbf{2}}$ & $\mathbf{\%}$ \\
\hline Cerradão/Cerrado/Mata/Reflorestamento & 287,30 & 20,94 \\
Campo limpo/Campo sujo/Campo cerrado & 469,46 & 34,21 \\
Pastagem & 477,48 & 34,79 \\
Agricultura & 130,94 & 9,54 \\
Influência urbana & 7,12 & 0,52 \\
\hline Total & $1.372,30$ & 100,00 \\
\hline
\end{tabular}

Elaboração: A autora.

No cenário real (ano de 2010) a bacia, conforme mapeamento de uso da terra e cobertura vegetal nativa, apresentava cerca de $44,85 \%$ de sua área coberta por classes de uso antrópico e $55,15 \%$ de sua área coberta, predominantemente, por classes de vegetação nativa.

Os dados da vazão diária máxima (Qmáx), mínima (Qmín) e média (Qméd), escoamento total anual (Qtot em mm/ano), para o cenário real (2010) e os cenários hipotéticos propostos, estão apresentados na Tabela III.

Tabela III - Vazão diária máxima, mínima e média, escoamento total anual para o ano de 2010 e diferentes cenários hipotéticos, na bacia hidrográfica do alto curso do rio Misericórdia - Dados MGB

\begin{tabular}{l|rrr|r}
\hline Cenário & $\begin{array}{r}\text { Qmáx } \\
\left(\mathbf{m}^{3} / \mathbf{s}\right)\end{array}$ & $\begin{array}{r}\text { Qmín } \\
\left(\mathbf{m}^{\mathbf{3}} / \mathbf{s}\right)\end{array}$ & $\begin{array}{r}\text { Qméd } \\
\left(\mathbf{m}^{3} / \mathbf{s}\right)\end{array}$ & $\begin{array}{r}\text { Qtot } \\
(\mathbf{m m} / \mathbf{a n o})\end{array}$ \\
\hline Cenário real (2010) & 69,54 & 8,47 & 22,77 & 523,17 \\
100\% Cobertura vegetal nativa & 76,27 & 10,18 & 23,98 & 551,04 \\
$100 \%$ Pastagem & 105,72 & 6,19 & 24,74 & 568,62 \\
100\% Agricultura & 47,28 & 9,25 & 19,24 & 442,25 \\
Conversão da pastagem para agricultura & 64,92 & 8,83 & 22,35 & 513,61 \\
\hline
\end{tabular}

Fonte: MGB. Elaboração: A autora.

A partir dos dados apresentados na Tabela III, verificou-se que, para o cenário real (2010), a vazão média foi de $22,77 \mathrm{~m}^{3} / \mathrm{s}$, com $523,17 \mathrm{~mm}$ de escoamento total anual para a bacia em estudo.

No que se refere aos cenários hipotéticos simulados, a mesma tabela mostrou que houve um aumento para a vazão média calculada para a bacia, nos cenários de 100\% Cobertura vegetal nativa e 100\% Pastagem, 
em relação ao ano de 2010. Para os cenários de 100\% Agricultura e Conversão da pastagem para agricultura, no entanto, verificou-se a redução da vazão média, para a mesma. Porém, em nenhuma das simulações hipotéticas realizadas houve alteração significativa em relação às vazões médias calculadas pelo modelo.

Collischonn (2001), simulou cenários hipotéticos semelhantes para a bacia do rio Taquari-Antas, no Rio Grande do Sul, e comparou os dados com a simulação do cenário real da bacia, do ano de 1995. Assim, o mesmo autor encontrou em seus estudos redução da vazão média e escoamento total anual para os cenários de $100 \%$ Floresta e aumento da mesma para os cenários de $100 \%$ Pastagem e 100\% cultura anual. Os valores do escoamento total anual foram: redução em $62 \mathrm{~mm} / \mathrm{ano}$, a partir do cenário 100\% Floresta, aumento de $31 \mathrm{~mm} /$ ano, no cenário de 100\% Pastagem e aumento de $34 \mathrm{~mm} /$ ano, no cenário de $100 \%$ cultura anual.

Considerando os dados simulados, no cenário hipotético que a bacia em estudo apresentou uma situação de $100 \%$ de Cobertura vegetal nativa, ocorreu um aumento das vazões máxima, mínima e média. Verificou-se um aumento de 27,87 mm (5,33\%) de escoamento total anual, aumento de $6,73 \mathrm{~m}^{3} / \mathrm{s}(9,68 \%)$ na vazão máxima, de $1,71 \mathrm{~m}^{3} / \mathrm{s}(20,19 \%)$ na vazão mínima e aumento de $1,21 \mathrm{~m}^{3} / \mathrm{s}(5,31 \%)$ na vazão média em comparação aos dados do ano de 2010.

No cenário hipotético em que a bacia apresentou uma situação de $100 \%$ de Pastagem, ocorreu um aumento da vazão máxima e média e redução da vazão mínima. Verificou-se um aumento de 45,45 mm $(8,69 \%)$ de escoamento total anual, aumento de $36,18 \mathrm{~m}^{3} / \mathrm{s}(52,03 \%)$ na vazão máxima, redução de 2,28 $\mathrm{m}^{3} / \mathrm{s}(26,92 \%)$ na vazão mínima e aumento em $1,97 \mathrm{~m}^{3} / \mathrm{s}(8,65 \%)$ na vazão média, na mesma comparação anterior.

No próximo cenário hipotético, em que bacia apresentou uma situação de $100 \%$ de Agricultura, ocorreu uma redução da vazão máxima e média e aumento da vazão mínima. Verificou-se uma redução de 80,92 mm $(15,47 \%)$ de escoamento total anual, redução de $22,26 \mathrm{~m}^{3} / \mathrm{s}(32,01 \%)$ na vazão máxima, aumento de $0,78 \mathrm{~m}^{3} / \mathrm{s}(9,21 \%)$ na vazão mínima e, novamente, redução em $3,53 \mathrm{~m}^{3} / \mathrm{s}(15,50 \%)$ na vazão média, ainda na mesma comparação com os dados do cenário real (2010).

Por fim, no último cenário simulado, ou seja, cenário onde ocorreu a conversão da pastagem para agricultura, houve também uma redução da vazão máxima e média e aumento da vazão mínima. Verificou-se uma redução de 9,56 mm (1,83\%) de escoamento total anual, redução de 4,62 m³ $(6,64 \%)$ na vazão máxima, aumento de $0,36 \mathrm{~m}^{3} / \mathrm{s}(4,25 \%)$ na vazão mínima e redução de $0,42 \mathrm{~m}^{3} / \mathrm{s}(1,84 \%)$ na vazão média, novamente em comparação aos dados de vazão do ano de 2010. 
De forma geral, a simulação da vazão bacia, a partir de cenários hipotéticos permitiu verificar que a substituição do uso da terra de $100 \%$ de cobertura vegetal nativa pelo uso da pastagem, aumentou a vazão máxima $\left(76,27\right.$ para $\left.105,72 \mathrm{~m}^{3} / \mathrm{s}\right)$ e a vazão média $\left(23,98\right.$ para $\left.24,74 \mathrm{~m}^{3} / \mathrm{s}\right)$ e reduziu a vazão mínima $\left(10,18\right.$ para $\left.6,19 \mathrm{~m}^{3} / \mathrm{s}\right)$. A substituição do uso da terra de $100 \%$ de cobertura vegetal nativa pelo uso da agricultura, por sua vez, resultou em redução da vazão máxima (76,27 para 47,28 m³ s), mínima (10,18 para $\left.9,25 \mathrm{~m}^{3} / \mathrm{s}\right)$ e da vazão média $\left(23,98\right.$ para $\left.19,24 \mathrm{~m}^{3} / \mathrm{s}\right)$.

\section{Considerações finais}

O sensoriamento remoto/geoprocessamento e a modelagem hidrológica permitiram, respectivamente, um maior e mais eficaz acesso/tratamento dos dados espaciais e a simulação de cenários hipotéticos para comparações dos dados de vazão e análise dos mesmos, de forma aprimorada e confiável.

Considerando todos os cenários hipotéticos simulados e a comparação com os dados de vazão calculados pelo MGB, para o cenário real de 2010, destacou-se que a vazão máxima aumentou para os cenários de $100 \%$ de Cobertura vegetal nativa (em $6,73 \mathrm{~m}^{3} / \mathrm{s}$ ) e $100 \%$ Pastagem (em 36,18 m³ $)$. Houve redução da vazão máxima para o cenário de $100 \%$ Agricultura $\left(\mathrm{em} 22,26 \mathrm{~m}^{3} / \mathrm{s}\right)$ e para o cenário de Conversão da pastagem para agricultura (em 4,62 $\left.\mathrm{m}^{3} / \mathrm{s}\right)$.

A vazão mínima aumentou para os cenários de $100 \%$ de Cobertura vegetal nativa (em $1,71 \mathrm{~m}^{3} / \mathrm{s}$ ), para o cenário de $100 \%$ Agricultura (em $0,78 \mathrm{~m}^{3} / \mathrm{s}$ ) e para o cenário de Conversão da pastagem para agricultura (em $0,36 \mathrm{~m}^{3} / \mathrm{s}$ ). Houve redução na vazão mínima apenas para o cenário de $100 \%$ Pastagem (em 2,28 m³/s).

A vazão média para os cenários hipotéticos propostos se manteve praticamente equiparada, com leve redução para o cenário de Conversão da pastagem para a agricultura (em $0,42 \mathrm{~m}^{3} / \mathrm{s}$ ) e aumento para os cenários de $100 \%$ de Cobertura vegetal nativa (em 1,21 m³ $/ \mathrm{s}$ ) e $100 \%$ Pastagem (em 1,77 m³ $/ \mathrm{s}$ ). A maior alteração da vazão média ocorreu me relação ao cenário de 100\% Agricultura, que teve redução em 3,53 $\mathrm{m}^{3} / \mathrm{s}$

O modelo utilizado foi definido com sensibilidade em relação às mudanças no uso e cobertura do solo na bacia considerada. Desta forma, a partir da simulação de cenários alternativos de mudanças no uso da terra e cobertura vegetal nativa, foi observado que o aumento da área agrícola na bacia resultou em uma diminuição da vazão média e, o aumento da área de pastagem, resultou em um aumento da vazão média para a mesma área de estudo.

Por fim, partindo-se das ferramentas escolhidas e simulações realizadas, verificou-se como as alterações do uso da terra e cobertura vegetal nativa poderiam influenciar a vazão dos cursos d'água presentes na 
área de estudo, evidenciando como a modelagem hidrológica pode auxiliar o planejamento da ocupação deste espaço da maneira mais adequada possível, em busca da manutenção da água presente na bacia hidrográfica.

\section{Referências}

ANA. Agência Nacional de Águas. Hidroweb - Sistema de Informações hidrológicas. Disponível em: <http:// hidroweb.ana.gov.br> Acesso em: mar. 2013.

BAYER, D. M.; COLLISCHONN, W. Análise de sensibilidade do Modelo MGB-IPH a mudanças de uso da terra. RBRH - Revista Brasileira de Recursos Hídricos. Vol. 18 n.3 - jul./set. 2013, p. 165-179.

CBH-ARAGUARI. Comitê da Bacia Hidrográfica do Rio Araguari. Disponível em:

<http://www.cbharaguari.org.br/>. Acesso em: mar. 2013.

CHAUDHRY, F. H.; PAIVA, J. B. D.; REIS, L. F. R. Monitoramento de Bacias Hidrográficas e Processamento de Dados/ Organizado por Fazal H. Chaudhry, João Batista Dias de Paiva; Luiza Fernanda Ribeiro Reis - São Carlos: RIMA, 2004. 326p.

CHRISTOFOLETTI, A. Modelagem de Sistemas Ambientais. São Paulo: Ed. Edgard Blücher Ltda., 1999. 236 p.

COLLISCHONN, W. Simulação hidrológica de grandes bacias. 2001. 270 f. Tese (Doutorado em Engenharia de Recursos Hídricos e Saneamento Ambiental) - Universidade Federal do Rio Grande do Sul, Porto Alegre, 2001.

RENNÓ, C. D.; SOARES, J. V. Conceitos básicos de modelagem hidrológica. In: MEIRELLES, M. S. P. Geomática: modelos e aplicações ambientais / Editores técnicos: Margareth Simões Penello Meirelles, Gilberto Câmara e Cláudia Maria de Almeida - Brasília: Embrapa Informação Tecnológica, 2007. 593 p.

ROCHA, J. S. M. Manual de manejo integrado de bacias hidrográficas. Santa Maria: Edições UFSM, 1991. $181 \mathrm{p}$.

ROSA, R. Estoque de Carbono em Solos sob Pastagens Cultivadas na Bacia Hidrográfica do Rio Paranaíba: Mapeamento e Modelagem. 2012. 198 f. Relatório (Pós-doutorado em Geociências Aplicadas) - Universidade de Brasília - Instituto de Geociências, Brasília, 2012.

TUNDISI, J. G.; TUNDISI, T. M. A água. São Paulo: Publifolha, 2005.

UFRGS. Universidade Federal do Rio Grande do Sul - Instituto de Pesquisas Hidráulicas. Hidrologia em grande escala. Disponível em: < https://www.ufrgs.br/hge/mgb-iph/> Acesso em: mar. 2011. 